\title{
Study of Widely Linear SC-FDE Systems for Interference Cancellation
}

\author{
Rogério Pereira Junior, Bruno Sens Chang and Carlos Aurélio Faria da Rocha
}

\begin{abstract}
In this paper, a performance analysis of widely linear (WL) equalizers for single carrier systems using frequency domain equalization (SC-FDE) under multiple independent interferences is presented. In this context, the expressions of the mean square error (MSE) and bit error rate (BER) for linear and WL equalizers in their regular and iterative versions for this scenario were derived. It was seen that the results obtained with these expressions match the simulated ones. They also show that the performance of the WL equalizers is better when compared to their linear versions regardless of the number of interferences.
\end{abstract}

Index Terms-SC-FDE systems, widely linear equalizers, interference cancellation.

\section{INTRODUCTION}

An alternative approach to the OFDM scheme, which allows to solve its peak to average power ratio (PAPR) problem in addition to maintain its low complexity frequency domain equalization, is the usage of single-carrier systems with frequency domain equalization (SC-FDE) [1] [2]. By using the cyclic prefix $(\mathrm{CP})$, the FDE is used at the receiver to compensate for the distortions imposed by the channel, and can achieve similar or even better performance than the OFDM scheme. For this reason, a hybrid solution was employed for the LTE cellular system; while in the downlink a OFDM-based scheme is used, a SC-FDE-based is employed in the uplink, where energy efficient power amplifiers in user terminals are required to improve battery life.

Besides the channel distortion problem, strong co-channel interference $(\mathrm{CCI})$ can arise in a variety of scenarios in a communications system, such as the case of a cellular system striving to obtain maximum spectral efficiency and using a frequency reuse factor of one [3], [4], [5]. In this type of situation, in addition to the desired signal, other signals on the same frequencies by unwanted transmitters can reach the receiver and lead to a drop in performance.

In order to cancel the harmful effects of these interferences, joint intersymbol interference (ISI) and CCI suppression schemes are required. Generally, minimum mean-squared

Rogério P. Junior is with Communications Research Group, Federal University of Santa Catarina, Florianópolis, Brazil, email:rogeriopereirajunior@gmail.com.

Bruno Sens Chang is with CPGEI/Electronics Department, Federal University of Technology - Paraná, Curitiba, Brazil, email:bschang@utfpr.edu.br.

Carlos A. Faria da Rocha is with Communications Research Group, Federal University of Santa Catarina, Florianópolis, Brazil, email:aurelio@eel.ufsc.br.

Digital Object Identifier: 10.14209/jcis.2019.8 error (MMSE) and decision feedback equalizers (DFE) are often used to suppress these harmful effects [6]. While the first equalizer minimizes the mean square error between the transmitted signal and the estimated signal, the DFE has a non-linear structure which uses a feedforward (FF) and a feedback (FB) filter. In general, the FF filter is a linear equalizer that aims to minimize the precursor ISI. The FB filter is used then to remove the ISI part which is left behind by FF filter. This structure can be hybrid (the FF filter in the frequency domain and the FB filter in the time domain [7]) or both filters can be realized in the frequency domain. This last structure can be implemented iteratively [8], leading to the known IB-DFE (Iterative Block Decision-Feedback Equalizer) scheme.

It is known that the use of DFE provides better performance against ISI when compared with the linear MMSE equalizer [9]. Thus, a natural question that arises is whether this performance improvement provided by the DFE will present itself in the co-channel interference cancellation process. In [10], the authors proposed the use of a iterative version of the DFE in SC-FDE systems in order to deal with the co-channel interference. The results showed that the proposed receiver can reduce the effect of interference in the estimated signal, clearly outperforming the conventional structure.

When the desired signal is provided by a real constellation like Binary Phase Shift Keying (BPSK) or Pulse Amplitude Modulation (PAM), its pseudo-correlation function is nonzero. Thus, the signal can be called improper, and the autocorrelation function alone is not enough to describe all its second-order statistics. This impropriety can be exploited at the receiver through widely linear (WL) processing [11] where the signal is used along with its conjugate version to obtain a more accurate estimate than when using strictly linear (SL) processing. In addition, for signals corrupted by one single improper interference, it has been demonstrated by the Single Antenna Interference Cancellation scheme (SAIC) proposed in [12], that widely linear receivers can drastically suppress the effect of this interference. Other approaches with the SAIC system for cancellation of interferences (ISI and CCI) were investigated in [13], [14].

Already in [15] and [16] the authors used a widely linear MMSE equalizer in the time domain, considering a scenario where we have more than one interference. It was shown in [15] that the performance of WL processing, in terms of bit error rate, decreases as the number of improper interferences 
increases. In [16], it has been seen that the WL equalizer will have a performance advantage with up to five interferences compared to its SL version.

Applications of WL equalizers in SC-FDE systems was also investigated in several works. In [17], a SC-FDE system using a widely linear equalizer over a MIMO (multiple-input multiple-output) channel and operating under a CCI was studied. The equalizer is designed to sample the received signal and then process the sampled block using a FF filter and another FB filter with noise prediction. Numerical results show that the usage of widely linear processing brings a performance gain even with a moderate block length. A widely linear version of the IB-DFE was proposed and studied in [18], [19] for SC-FDE systems without the presence of interfering signals. It was found out that it has a better error performance and a higher robustness to channel estimation errors when compared to the SL version. Finally in [20] and [21], the authors proposed a WL-MMSE and WL-IB-DFE equalizer respectively for a SC-FDE system under the effect of up to $K$ known interferences. It was seen that the system becomes more robust in terms of BER applying the widely linear equalizers with respect to its SL versions.

The goal of the present work is to develop analytical expressions for the MSE for the widely linear equalizers presented in [20], [21] in order to obtain the signal-tointerference-plus-noise ratio (SINR) and consequently the analytical BER for the MMSE and IB-DFE equalizers in their strictly and widely linear versions. Results obtained with these expressions were verified and analyzed with respect to the simulation results and the behavior of the system when the number of interferences grows respectively. It was found out that the results obtained from the derived equations match the ones obtained from simulations in a variety of scenarios. With these equations validated, a MSE analysis is also made, in which it is possible to see that the WL SC-FDE system, mainly with the iterative equalizer, will always have a better error performance that the linear version, regardless of the number of interferences. The main contribution of this work is to present a well-developed theoretical analysis for the WL-MMSE and WL-IB-DFE equalizers in an SCFDE system with multiple interferences, besides illustrating the superior performance of both equalizers in suppressing these interferences (ISI and CCI) compared to conventional equalizers.

The remainder of this paper is organized as follows: Section III presents the widely linear MMSE and widely linear iterative equalizers for SC-FDE systems considered in this paper. In Section [III, the expressions of the SINR at the output of the receiver for the proposed system model are derived, whereas simulation results showing the precision of these expressions are shown in Section IV Finally, the concluding remarks are presented in Section $\mathrm{V}$.

In this paper, the vectors and matrices in the frequency domain are represented respectively by lowercase letters and capital letters both in bold. Time domain elements have a tilde appended to them. The superscripts $(). *(.)^{T}$,
$(.)^{H}$ denote, respectively, conjugate, transpose and Hermitian operations. The $i$-th element of the vector $\mathbf{v}$ is given by $v_{i}$. The identity matrix of size $N \times N$ is denoted by $\mathbf{I}_{N}$. The trace of a matrix $\mathbf{M}$ is expressed by $\operatorname{Tr}[\mathbf{M}]$. Finally, the mathematical expectation is $\mathrm{E}\{$.$\} .$

\section{System Model}

For all considered systems, the information block $\tilde{\mathbf{s}}$, with power $\sigma_{s}^{2}$ considered as unity and size (samples) $N$, is transmitted in parallel with $K$ interfering signals with the same structure, represented by $\tilde{\mathbf{s}}_{i k}$ and with power $\sigma_{i}^{2}$. Without loss of generality, all the signals come from an improper constellation (in our case, BPSK). In order to eliminate the Interblock Interference (IBI), both useful and interfering signals add a $L_{C P}$-sized cyclic prefix to the transmission by parallel channels (all of size $L$ ). Before the entry of the receiver, the combination of useful and interference signals is also corrupted by noise. Thus, the vector received after the removal of the cyclic prefix can be written as

$$
\tilde{\mathbf{r}}=\tilde{\mathbf{H}} \tilde{\mathbf{s}}+\sum_{k=0}^{K} \tilde{\mathbf{H}}_{i k} \tilde{\mathbf{s}}_{i k}+\tilde{\mathbf{n}},
$$

where $\tilde{\mathbf{H}}$ and $\tilde{\mathbf{H}}_{i k}$ represent respectively the effect of the main channel and the interfering signals, and $\tilde{\mathbf{n}}$ is a White Gaussian noise vector with zero mean and variance $\sigma_{n}^{2}$ and length $N+L_{C P}$. The absence of the IBI after CP removal is guaranteed, since $L_{C P}$ is greater than or equal to the delay spread of the channel. Due to the insertion of the cyclic prefix the channel matrix $\tilde{\mathbf{H}}$ is circulant, with its first column containing the channel impulse response $\tilde{\mathbf{h}}$ appended by $N-$ $L$ zeros. Hence this matrix has the following decomposition:

$$
\tilde{\mathbf{H}}=\mathbf{W}^{H} \mathbf{H W},
$$

where $\mathbf{W}$ is the normalized discrete Fourier transform (DFT) matrix with size $N \times N$ and $\mathbf{H}$ is a $N \times N$ diagonal matrix corresponding to the channel frequency response $\tilde{\mathbf{h}}$. The same procedure is done for the interference channels $\tilde{\mathbf{H}}_{i k}$, leading to $\mathbf{W}^{H} \mathbf{H}_{i k} \mathbf{W}$. The frequency-domain representation of the received signal can be thus written as [20]:

$$
\mathbf{r}=\mathbf{W} \tilde{\mathbf{r}}=\mathbf{H s}+\sum_{k=0}^{K} \mathbf{H}_{i k} \mathbf{s}_{i k}+\mathbf{n},
$$

where $\mathbf{s}=\mathbf{W} \tilde{\mathbf{s}}$ is the desired transmitted signal in the frequency domain, $\mathbf{s}_{i k}$ is the frequency domain signal from the $k$-th interference and $\mathbf{n}=\mathbf{W} \tilde{\mathbf{n}}$ is the frequency domain noise. The corresponding signal-to-interference ratio (SIR) can be then given as $\frac{\sigma_{s}^{2}}{\sigma_{i}^{2}}$. Finally, the signal is passed through the widely linear equalizer $\mathbf{A}$ and the output vector is transformed to the time domain through an IFFT for symbol detection.

\section{A. WL-MMSE equalizer}

The block diagram of the considered SC-FDE system with widely linear equalization is given in Figure 1 


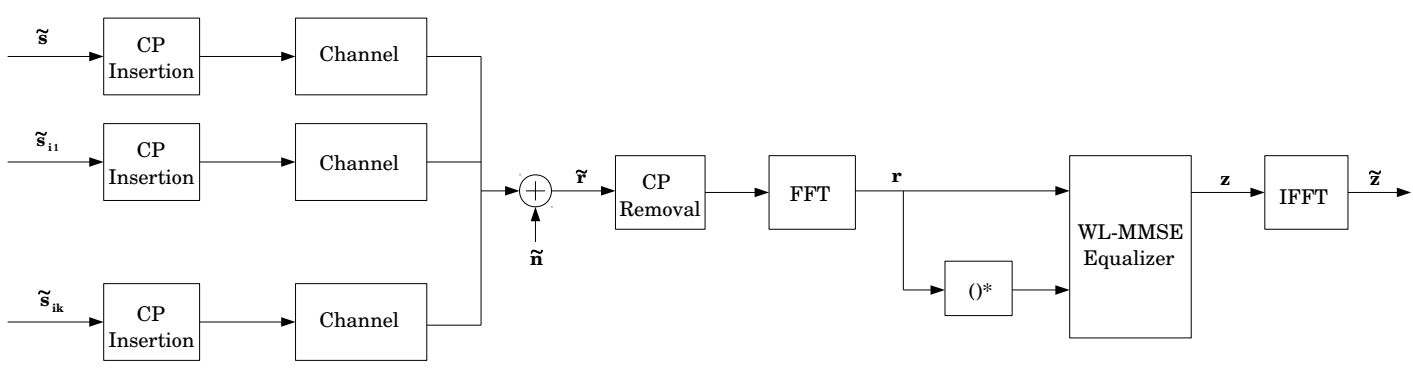

Figure 1. A SC-FDE system employing widely linear MMSE equalization under the effect of $K$ interferences.

Since we are transmitting a real signal through a complex channel, the pseudo-covariance of the resulting signal is nonzero. Therefore, it is possible use advantageously widely linear processing, processing $\mathbf{r}$ and $\mathbf{r}^{*}$ together to determine the equalizer in order to obtain an improvement in signal estimation and interference cancellation. Applying these concepts to the considered system, the signal at the output of the equalizer $\mathbf{z}$, with size $N$, is given by $\mathbf{A}^{H} \mathbf{t}$, where $\mathbf{A}^{H}$ is of size $N \times 2 N$ and $\mathbf{t}=\left[\begin{array}{l}\mathbf{r} \\ \mathbf{r}^{*}\end{array}\right]$ is a vector with length $2 N$.

The mean square error between the estimated $\operatorname{signal} \mathbf{z}$ and the desired signal $\mathbf{s}$ is given by:

$$
\begin{aligned}
\epsilon_{w l} & =E\left\{\left[\left|\mathbf{A}^{H} \mathbf{t}-\mathbf{s}\right|^{2}\right]\right\}=E\left\{\left[\left(\mathbf{t}^{H} \mathbf{A}-\mathbf{s}^{H}\right)\left(\mathbf{A}^{H} \mathbf{t}-\mathbf{s}\right)\right]\right\} \\
& =\operatorname{Tr}\left[\mathbf{A}^{H} \mathbf{C}_{\mathbf{t t}} \mathbf{A}-\mathbf{A}^{H} \mathbf{C}_{\mathbf{t s}}-\mathbf{C}_{\mathbf{s t}} \mathbf{A}+\mathbf{I}_{N}\right],
\end{aligned}
$$

where

$$
\begin{gathered}
\mathbf{C}_{\mathbf{t t}}=E\left\{\mathbf{t t}^{H}\right\}=E\left\{\left[\begin{array}{c}
\mathbf{r} \\
\mathbf{r}^{*}
\end{array}\right]\left[\begin{array}{ll}
\mathbf{r}^{H} & \mathbf{r}^{T}
\end{array}\right]\right\}=\left[\begin{array}{cc}
\mathbf{C}_{\mathbf{r r}} & \overline{\mathbf{C}}_{\mathbf{r r}} \\
\overline{\mathbf{C}}_{\mathbf{r r}}^{*} & \mathbf{C}_{\mathbf{r r}}^{*}
\end{array}\right] \\
\mathbf{C}_{\mathbf{r r}}=E\left\{\mathbf{r r}^{H}\right\}=\mathbf{H H}^{H}+\sigma_{i}^{2} \sum_{k=0}^{K} \mathbf{H}_{i k} \mathbf{H}_{i k}^{H}+\sigma_{n}^{2} \mathbf{I}_{N}, \\
\overline{\mathbf{C}}_{\mathbf{r r}}=E\left\{\mathbf{r r}^{T}\right\}=\mathbf{H} \mathbf{U H}^{T}+\sigma_{i}^{2} \sum_{k=0}^{K} \mathbf{H}_{i k} \mathbf{U H}_{i k}^{T}, \\
\mathbf{C}_{\mathbf{t s}}=E\left\{\mathbf{t s}^{H}\right\}=\mathbf{C}_{\mathbf{s t}}^{\mathbf{H}}=\left[\begin{array}{c}
\mathbf{H} \\
\mathbf{H}^{*} \mathbf{U}
\end{array}\right],
\end{gathered}
$$

and

$$
\mathbf{C}_{\mathbf{s t}}=E\left\{\mathbf{s t}^{H}\right\}=\mathbf{C}_{\mathbf{t s}}^{\mathbf{H}}=\left[\begin{array}{ll}
\mathbf{H}^{H} & \mathbf{H}^{T} \mathbf{U}
\end{array}\right]
$$

with $\mathbf{U}=\mathbf{W W}^{\mathbf{T}}$ expressed by

$$
\mathbf{U}=\left[\begin{array}{ccccc}
1 & 0 & 0 & \ldots & 0 \\
0 & 0 & 0 & \ldots & 1 \\
\vdots & \vdots & \vdots & \vdots & \vdots \\
0 & 0 & 1 & \ldots & 0 \\
0 & 1 & 0 & \ldots & 0
\end{array}\right],
$$

Thus, using the minimum mean square error (MMSE) criterion, the widely linear equalizer $\mathbf{A}$ is given by:

$$
A=\mathbf{C}_{\mathrm{tt}}^{-1} \mathbf{C}_{\mathrm{ts}} \text {. }
$$

For comparison, the strictly linear MMSE equalizer $\mathbf{A}_{L}=$ $\mathbf{C}_{\mathbf{r r}}^{-1} \mathbf{C}_{\mathbf{r s}}$ of size $N \times N$ can be given as

$$
\mathbf{A}_{L}=\left(\mathbf{H} \mathbf{H}^{H}+\sigma_{i}^{2} \sum_{k=0}^{K} \mathbf{H}_{i k} \mathbf{H}_{i k}^{H}+\sigma_{n}^{2} \mathbf{I}_{N}\right)^{-1} \mathbf{H} .
$$

\section{B. WL-MMSE IB-DFE equalizer}

The block diagram of the considered SC-FDE system with widely linear iterative equalization is presented in Figure 2 The proposed iterative equalizer, which is used to cancel the known interferences and obtain the symbol estimate, consists in a feedforward filter and in a feedback one, both operating in the frequency domain. Thus, the estimated symbol vector $\hat{\mathbf{s}}^{l}$ for the $l$-th iteration is formed by

$$
\hat{\mathbf{s}}^{l}=\mathbf{y}^{\prime}, l+\mathbf{y}^{\prime \prime}, l,
$$

where $\mathbf{y}^{\prime}, l$ is the output of the feedforward filter in the $l$-th iteration and is given by

$$
\mathbf{y}^{\prime}, l=\mathbf{A}^{l, H} \mathbf{t},
$$

with $\mathbf{A}^{l, H}$ is a matrix corresponding to the feedforward filter of size $N \times 2 N$ and $\mathbf{t}=\left[\begin{array}{c}\mathbf{r} \\ \mathbf{r}^{*}\end{array}\right]$ again is the vector with length $2 N$ that encompasses the received signal and its conjugate. The feedforward filter aim is to maximize the SINR of the detected symbols at every iteration. The vector $\mathbf{y}^{\prime \prime}, l$ is the output of the feedback filter, and it can be expressed as

$$
\mathbf{y}^{\prime \prime}, l=\mathbf{B}^{l, H} \hat{\mathbf{s}}^{l-1},
$$

where $\mathbf{B}^{l, H}$ is the $N \times N$ matrix corresponding to the feedback filter and $\hat{\mathbf{s}}^{l-1}$ is the frequency domain estimated vector after symbol decision. The role of this feedback filter is the partial removal of the postcursor components of the ISI.

Both $\hat{\mathbf{s}}^{l}$ and $\hat{\mathbf{s}}^{l-1}$ are assumed to be independent and identically distributed (iid), with zero mean and no statistical relation to the noise. Thus, to obtain the coefficients for both the feedforward and the feedback filter at each iteration, the goal is to minimize the MSE in the following way:

$$
\begin{aligned}
\epsilon_{w l}^{l}= & E\left\{\left|\mathbf{A}^{l, H} \mathbf{t}+\mathbf{B}^{l, H} \hat{\mathbf{s}}^{l-1}-\mathbf{s}\right|^{2}\right\} \\
= & \operatorname{Tr}\left[\mathbf{A}^{l, H} \mathbf{C}_{\mathbf{t t}} \mathbf{A}^{l}+\mathbf{A}^{l, H} \mathbf{C}_{\mathbf{t} \hat{\mathbf{s}}} \mathbf{B}^{l}-\mathbf{A}^{l, H} \mathbf{C}_{\mathbf{t s}}\right. \\
& +\mathbf{B}^{l, H} \mathbf{C}_{\hat{\mathbf{s}} \mathbf{t}} \mathbf{A}^{l}+\mathbf{B}^{l, H} \mathbf{C}_{\hat{\mathbf{s}} \mathbf{s}} \mathbf{B}^{l}-\mathbf{B}^{l, H} \mathbf{C}_{\hat{\mathbf{s}} \mathbf{s}} \\
& \left.-\mathbf{C}_{\mathbf{s} \mathbf{t}} \mathbf{A}^{l}-\mathbf{C}_{\mathbf{s} \mathbf{s}} \mathbf{B}^{l}+\mathbf{I}_{N}\right],
\end{aligned}
$$




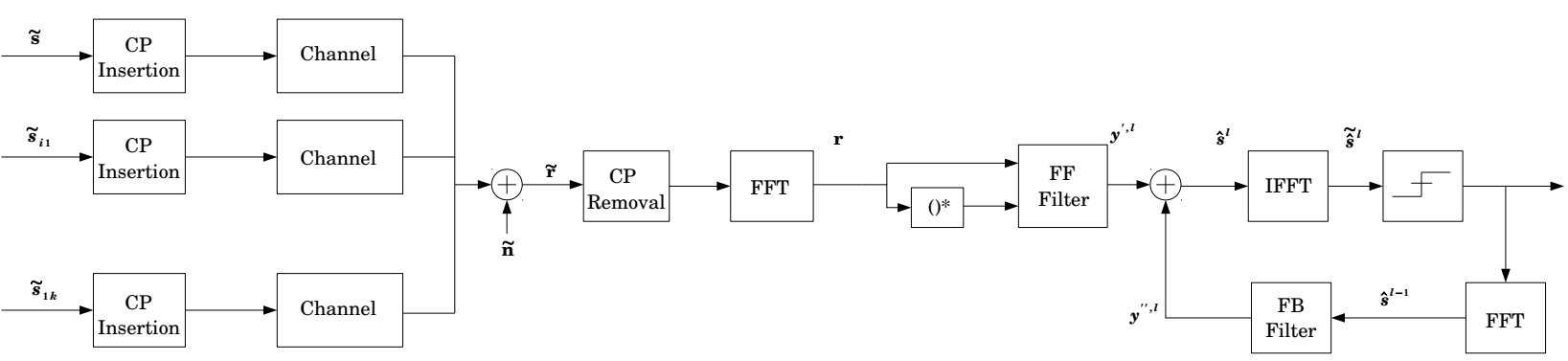

Figure 2. A SC-FDE system employing widely linear iterative MMSE equalization under the effect of $K$ interferers.

where

$$
\begin{aligned}
& \mathbf{C}_{\mathbf{t} \hat{\mathbf{s}}}= E\left\{\mathbf{t} \hat{\mathbf{s}}^{l-1, H}\right\}=E\left\{\left[\begin{array}{c}
\mathbf{r} \\
\mathbf{r}^{*}
\end{array}\right] \hat{\mathbf{s}}^{l-1, H}\right\} \\
&= {\left[\begin{array}{c}
\mathbf{H} E\left\{\hat{\mathbf{s}}^{l-1, H}\right\} \\
\mathbf{H} E\left\{\mathbf{s}^{*} \hat{\mathbf{s}}^{l-1, H}\right\}
\end{array}\right] } \\
&= {\left[\begin{array}{c}
\mathbf{H} \mathbf{C}_{\mathbf{s} \hat{\mathbf{s}}} \\
\mathbf{H}^{*} \mathbf{U} \mathbf{C}_{\mathbf{s}} \hat{\mathbf{s}}
\end{array}\right] } \\
& \mathbf{C}_{\mathbf{s} \hat{\mathbf{s}}}=E\left\{\mathbf{s}^{l-1, H}\right\}, \\
& \mathbf{C}_{\hat{\mathbf{s}} \mathbf{s}}=E\left\{\hat{\mathbf{s}}^{l-1} \mathbf{s}^{H}\right\} \\
&=\mathbf{C}_{\mathbf{s} \hat{\mathbf{s}}}^{H}, \\
& \mathbf{C}_{\hat{\mathbf{s}} \hat{\mathbf{s}}}=E\left\{\hat{\mathbf{s}}^{l-1} \hat{\mathbf{s}}^{l-1, H}\right\},
\end{aligned}
$$

and

$$
\begin{aligned}
& \mathbf{C}_{\hat{\mathbf{s}} \mathbf{t}}=E\left\{\hat{\mathbf{s}}^{l-1} \mathbf{t}^{H}\right\}=E\left\{\hat{\mathbf{s}}^{l-1}\left[\begin{array}{ll}
\mathbf{r}^{H} & \mathbf{r}^{T}
\end{array}\right]\right\} \\
& =\left[\begin{array}{ll}
\mathbf{H}^{H} E\left\{\hat{\mathbf{s}}^{l-1} \mathbf{s}^{H}\right\} & \mathbf{H}^{T} E\left\{\hat{\mathbf{s}}^{l-1} \mathbf{s}^{T}\right\}
\end{array}\right] \\
& =\left[\begin{array}{ll}
\mathbf{H}^{H} \mathbf{C}_{\hat{\mathbf{s} s}} & \mathbf{H}^{T} \mathbf{U} \mathbf{C}_{\hat{\mathrm{s} s}}
\end{array}\right] \\
& =\mathbf{C}_{\mathbf{t} \hat{\mathbf{s}}}{ }^{H} \text {, }
\end{aligned}
$$

The constraint that the feedback filter removes precursor and postcursor ISI, but not the desired symbol, is also imposed in this process, i.e. [22],

$$
\operatorname{Tr}\left(\mathbf{B}^{l}\right)=0 \text {. }
$$

Using the Lagrange multiplier method with respect to the feedback filter $\mathbf{B}^{l}$ to minimize the MSE presented in (16) under the constraint imposed by 23, we obtain

$$
\begin{aligned}
f\left(\mathbf{A}^{l}, \mathbf{B}^{l}, \lambda^{l}\right)= & \operatorname{Tr}\left[\mathbf{A}^{l, H} \mathbf{C}_{\mathbf{t t}} \mathbf{A}^{l}+\mathbf{A}^{l, H} \mathbf{C}_{\mathbf{t} \hat{\mathbf{s}}} \mathbf{B}^{l}\right. \\
& -\mathbf{A}^{l, H} \mathbf{C}_{\mathbf{t s}}+\mathbf{B}^{l, H} \mathbf{C}_{\hat{\mathbf{s}} \mathbf{t}} \mathbf{A}^{l}-\mathbf{B}^{l, H} \mathbf{C}_{\hat{\mathbf{s}} \mathbf{s}} \\
& +\mathbf{B}^{l, H} \mathbf{C}_{\hat{\mathbf{s}} \hat{\mathbf{s}}} \mathbf{B}^{l}-\mathbf{C}_{\mathbf{s t}} \mathbf{A}^{l}-\mathbf{C}_{\mathbf{s} \hat{\mathbf{s}}} \mathbf{B}^{l}+\mathbf{I}_{N} \\
& \left.+\lambda^{l} \mathbf{B}^{l}\right],
\end{aligned}
$$

where $\lambda^{l}$ is the Lagrange multiplier. By setting the gradient of (24) to zero with respect to $\mathbf{A}^{l, H}, \mathbf{B}^{l, H}$ and $\lambda^{l}$, respectively, we obtain

$$
\frac{\partial f\left(\mathbf{A}^{l}, \mathbf{B}^{l}, \lambda^{l}\right)}{\partial \mathbf{A}^{l, H}}=\mathbf{C}_{\mathbf{t t}} \mathbf{A}^{l}+\mathbf{C}_{\mathbf{t s}} \mathbf{B}^{l}-\mathbf{C}_{\mathbf{t s}}=0,
$$

$$
\frac{\partial f\left(\mathbf{A}^{l}, \mathbf{B}^{l}, \lambda^{l}\right)}{\partial \mathbf{B}^{l, H}}=\mathbf{C}_{\hat{\mathbf{s}}} \mathbf{A}^{l}+\mathbf{C}_{\hat{\mathbf{s}} \mathbf{s}} \mathbf{B}^{l}-\mathbf{C}_{\hat{\mathbf{s}} \mathbf{s}}+\lambda^{l} \mathbf{I}_{N}=0
$$

and

$$
\frac{\partial f\left(\mathbf{A}^{l}, \mathbf{B}^{l}, \lambda^{l}\right)}{\partial \lambda^{l}}=\operatorname{Tr}\left(\mathbf{B}^{l}\right)=0 .
$$

Developing 26, we can obtain an expression for $\mathbf{B}^{l}$, which can be given as

$$
\mathbf{B}^{l}=-\left(\mathbf{C}_{\hat{\mathbf{s}} \hat{\mathbf{s}}}\right)^{-1}\left[\mathbf{C}_{\hat{\mathbf{s}} \mathbf{t}} \mathbf{A}^{l}-\mathbf{C}_{\hat{\mathbf{s}} \mathbf{s}}+\lambda^{l} \mathbf{I}_{N}\right] .
$$

The value of $\lambda^{l}$ is calculated to uphold the restriction imposed by (23). With this information, the equation for $\mathbf{B}^{l}$ can now be rewritten as

$$
\mathbf{B}^{l}=-\left(\mathbf{C}_{\hat{\mathbf{s}} \hat{\mathbf{s}}}\right)^{-1}\left[\mathbf{C}_{\hat{\mathbf{s}} \mathbf{t}} \mathbf{A}^{l}-\Delta\right],
$$

where $\Delta$ is given by

$$
\Delta=\mathbf{C}_{\hat{\mathbf{s}} \mathbf{s}}+\frac{\operatorname{Tr}\left[\mathbf{C}_{\hat{\mathbf{s}} \mathbf{t}} \mathbf{A}^{l}\right]-\operatorname{Tr}\left[\mathbf{C}_{\hat{\mathbf{s} s}}\right]}{N} \mathbf{I}_{N}
$$

and

$$
\lambda^{l}=\frac{\operatorname{Tr}\left[\mathbf{C}_{\hat{\mathbf{s}} \mathbf{t}} \mathbf{A}^{l}\right]-\operatorname{Tr}\left[\mathbf{C}_{\hat{\mathbf{s}} \mathbf{s}}\right]}{N} .
$$

With this value of $\mathbf{B}^{l}$ we can now obtain the updated coefficients for $\mathbf{A}^{l}$, that can be expressed as

$$
\mathbf{A}^{l}=\mathbf{C}_{\mathrm{tt}}^{-1}\left(\mathbf{C}_{\mathrm{ts}}-\mathbf{C}_{\mathrm{ts}} \mathbf{B}^{l}\right) .
$$

We remind that at the first iteration $(l=1) \mathbf{B}^{1}=0$, due to the lack of previous decisions. $\mathbf{A}^{1}$ is also reduced to

$$
\mathbf{A}^{1}=\mathbf{C}_{\mathrm{tt}}^{-1} \mathbf{C}_{\mathrm{ts}},
$$

i.e., the WL-MMSE equalizer presented in II-A.

For every following iteration, the values of $\mathbf{A}^{l}$ and $\mathbf{B}^{l}$ are calculated according to (29) and (32). These iterations usually use the power of the previously detected symbols and the correlation between the received and the detected symbol vectors in order to improve the detection performance.

When perfect knowledge of the data is available, the feedforward filter $\mathbf{A}^{l}$ becomes the widely linear matched 
filter for the channel in order to maximize the signal-tonoise ratio (SNR), while the feedback filter removes all the ISI. However, the precise calculation of the correlation coefficient and the detected symbol power at each iteration of the equalizer can be a complex process [22].

In order to simplify this process, we assume that we have ideal feedback after the first iteration. Consequently, $\mathbf{C}_{\mathrm{s} \hat{\mathbf{s}}}=$ $\mathbf{C}_{\hat{\mathbf{s}} \hat{\mathbf{s}}}=\mathbf{C}_{\hat{\mathbf{s}} \mathbf{s}}=\sigma_{s}^{2} \mathbf{I}_{N}, \mathbf{C}_{\mathbf{t} \hat{\mathbf{s}}}=\mathbf{C}_{\mathbf{t s}}$ and $\mathbf{C}_{\hat{\mathbf{s}} \mathbf{t}}=\mathbf{C}_{\mathbf{s t}}$. Thus, 29] and (32) can now be rewritten as follows:

$$
\mathbf{A}^{l}=\mathbf{C}_{\mathbf{t t}}^{-\mathbf{1}}\left(\mathbf{I}_{N}-\mathbf{B}^{l}\right) \mathbf{C}_{\mathbf{t s}}
$$

and

$$
\mathbf{B}^{l}=-\left[\mathbf{C}_{\mathbf{s t}} \mathbf{A}^{l}-\rho \mathbf{I}_{N}\right],
$$

where $\rho$ is given by

$$
\rho=\frac{\operatorname{Tr}\left[\mathbf{C}_{\mathbf{s t}} \mathbf{A}^{l}\right]}{N} .
$$

With this, immediately after the first iteration the feedforward filter is switched to the WL matched filter. This assumption is feasible due to the good performance of the WL feedforward filter in the first iteration, providing the following ones with reliable symbol estimates. For comparison, the optimal coefficients for filters $\mathbf{A}_{L}^{l}$ and $\mathbf{B}_{L}^{l}$ for the SL case considering also that the decisions are correct are given by

$$
\mathbf{A}_{L}^{l}=\mathbf{C}_{\mathbf{r r}}^{-1}\left(\mathbf{I}_{N}-\mathbf{B}_{L}^{l}\right) \mathbf{C}_{\mathbf{r s}},
$$

and

$$
\mathbf{B}_{L}^{l}=-\left[\mathbf{C}_{\mathbf{s r}} \mathbf{A}_{L}^{l}-\eta \mathbf{I}_{N}\right]
$$

where $\eta=\frac{\operatorname{Tr}\left[\mathbf{C}_{\mathbf{s r}} \mathbf{A}_{L}^{l}\right]}{N}, \mathbf{C}_{\mathbf{r s}}=\mathbf{H}$ and $\mathbf{C}_{\mathbf{s r}}=\mathbf{H}^{H}$.

\section{ERROR PERFORMANCE ANALYSIS}

In this section, SINR and MSE expressions for all of the already presented equalizers will be derived.

\section{A. SINR for the Linear MMSE receiver with $K$ interferences}

After equalization and interference cancellation, an inverse FFT is done on $\mathbf{z}_{L}$ so that symbol decision is done on the time domain. Thus, the symbol estimate $\tilde{\mathbf{z}}_{L}$ (with size $N$ ) is expressed as

$$
\begin{aligned}
\tilde{\mathbf{z}}_{L}= & \mathbf{W}^{H} \mathbf{z}_{L}=\mathbf{W}^{H} \mathbf{A}_{L}^{H} \mathbf{r} \\
= & \mathbf{W}^{H}\left[\mathbf{A}_{L}^{H}\left(\mathbf{H} \mathbf{s}+\sum_{k=0}^{K} \mathbf{H}_{i k} \mathbf{s}_{i k}+\mathbf{n}\right)\right] \\
= & \mathbf{W}^{H}\left(\mathbf{A}_{L}^{H} \mathbf{H}\right) \mathbf{W} \tilde{\mathbf{s}}+\mathbf{W}^{H}\left(\mathbf{A}_{L}^{H} \sum_{k=0}^{K} \mathbf{H}_{i k}\right) \mathbf{W} \tilde{\mathbf{s}}_{i k}+ \\
& +\mathbf{W}^{H} \mathbf{A}_{L}^{H} \mathbf{n} .
\end{aligned}
$$

Combining the effect of ISI and noise, we can write the error vector $\tilde{\mathbf{e}}_{L}=\tilde{\mathbf{z}}_{L}-\tilde{\mathbf{s}}$ as

$$
\begin{aligned}
\tilde{\mathbf{e}}_{L}= & \mathbf{W}^{H}\left(\mathbf{A}_{L}^{H} \mathbf{H}-\mathbf{I}_{N}\right) \mathbf{W} \tilde{\mathbf{s}} \\
& +\mathbf{W}^{H}\left(\mathbf{A}_{L}^{H} \sum_{k=0}^{K} \mathbf{H}_{i k}\right) \mathbf{W} \tilde{\mathbf{s}}_{i k}+\mathbf{W}^{H} \mathbf{A}_{L}^{H} \mathbf{n} .
\end{aligned}
$$

The mean square error $\mathbf{J}\left(\tilde{\mathbf{e}}_{L}\right)=\operatorname{Tr}\left[\mathrm{E}\left\{\tilde{\mathbf{e}}_{L} \tilde{\mathbf{e}}_{L}^{H}\right\}\right]$ can be expressed for all $N$ block elements as

$$
\begin{aligned}
\mathbf{J}\left(\tilde{\mathbf{e}}_{L}\right)= & \operatorname{Tr}\left[\mathbf{W}^{\mathbf{H}}\left(\mathbf{A}_{L}^{H} \mathbf{H}-\mathbf{I}_{N}\right)\left(\mathbf{H}^{H} \mathbf{A}_{L}-\mathbf{I}_{N}\right) \mathbf{W}\right. \\
& +\mathbf{W}^{H} \sigma_{i}^{2}\left(\mathbf{A}_{L}^{H} \sum_{k=0}^{K} \mathbf{H}_{i k}\right)\left(\sum_{k=0}^{K} \mathbf{H}_{i k}^{H} \mathbf{A}_{L}\right) \mathbf{W} \\
& \left.+\sigma_{n}^{2} \mathbf{W}^{H}\left(\mathbf{A}_{L}^{H} \mathbf{A}_{L}\right) \mathbf{W}\right],
\end{aligned}
$$

where

$$
\begin{aligned}
\left(\mathbf{A}_{L}^{H} \mathbf{H}-\mathbf{I}_{N}\right)\left(\mathbf{H}^{H} \mathbf{A}_{L}-\mathbf{I}_{N}\right) & =\left(\sigma_{i}^{2} \sum_{k=0}^{K} \mathbf{H}_{i k} \mathbf{H}_{i k}^{H}+\sigma_{n}^{2} \mathbf{I}_{N}\right)^{2} \\
& \times\left(\mathbf{H H}^{H}+\sigma_{i}^{2} \sum_{k=0}^{K} \mathbf{H}_{i k} \mathbf{H}_{i k}^{H}+\sigma_{n}^{2} \mathbf{I}_{N}\right)^{-2}
\end{aligned}
$$

$$
\begin{array}{r}
\left(\mathbf{A}_{L}^{H} \sum_{k=0}^{K} \mathbf{H}_{i k}\right)\left(\sum_{k=0}^{K} \mathbf{H}_{i k}^{H} \mathbf{A}_{L}\right)=\left(\mathbf{H}^{H} \mathbf{H} \sum_{k=0}^{K} \mathbf{H}_{i k} \mathbf{H}_{i k}^{H}\right) \\
\times\left(\mathbf{H H}^{H}+\sigma_{i}^{2} \sum_{k=0}^{K} \mathbf{H}_{i k} \mathbf{H}_{i k}^{H}+\sigma_{n}^{2} \mathbf{I}_{N}\right)^{-2}
\end{array}
$$

$$
\begin{aligned}
\mathbf{A}_{L}^{H} \mathbf{A}_{L}= & \left(\mathbf{H H}^{H}+\sigma_{i}^{2} \sum_{k=0}^{K} \mathbf{H}_{i k} \mathbf{H}_{i k}^{H}+\sigma_{n}^{2} \mathbf{I}_{N}\right)^{-2} \\
& \times\left(\mathbf{H H}^{H}\right) .
\end{aligned}
$$

Based on the previous equations, we can rewrite 41 as

$$
\begin{aligned}
\mathbf{J}\left(\tilde{\mathbf{e}}_{L}\right)= & \operatorname{Tr}\left[\left(\mathbf{H} \mathbf{H}^{H}+\sigma_{i}^{2} \sum_{k=0}^{K} \mathbf{H}_{i k} \mathbf{H}_{i k}^{H}+\sigma_{n}^{2} \mathbf{I}_{N}\right)^{-2}\right. \\
& \times\left(\sigma_{i}^{2} \sum_{k=0}^{K} \mathbf{H}_{i k} \mathbf{H}_{i k}^{H}+\sigma_{n}^{2} \mathbf{I}_{N}\right)^{2} \\
& \left.+\mathbf{H}^{H} \mathbf{H}\left(\sigma_{i}^{2} \sum_{k=0}^{K} \mathbf{H}_{i k} \mathbf{H}_{i k}^{H}+\sigma_{n}^{2} \mathbf{I}_{N}\right)\right]
\end{aligned}
$$

Thus, we can express the mean square error for all $N$ block elements at the output of the equalizer as

$$
\begin{aligned}
\mathbf{J}\left(\tilde{\mathbf{e}}_{L}\right)= & \frac{1}{N} \operatorname{Tr}\left[\left(\mathbf{H H}^{H}+\sigma_{i}^{2} \sum_{k=0}^{K} \mathbf{H}_{i k} \mathbf{H}_{i k}^{H}+\sigma_{n}^{2} \mathbf{I}_{N}\right)^{-1}\right. \\
& \left.\times\left(\sigma_{i}^{2} \sum_{k=0}^{K} \mathbf{H}_{i k} \mathbf{H}_{i k}^{H}+\sigma_{n}^{2} \mathbf{I}_{N}\right)\right]
\end{aligned}
$$


Removing the bias from the impartial estimate, the instantaneous SINR $\gamma_{M M S E}$ at the output of the equalizer can be expressed as [23].

$$
\gamma_{M M S E}=\frac{1}{\mathbf{J}\left(\tilde{\mathbf{e}}_{L}\right)}-1 .
$$

\section{B. SINR for the WL-MMSE receiver with $K$ interferences}

We recall that after the FFT, the received signal and its conjugate version are grouped in the vector $\mathbf{t}$. Both versions are processed together in the frequency domain by the WLMMSE equalizer A. Thus, the symbol estimate $\tilde{\mathbf{z}}$ in the time domain is expressed by

$$
\tilde{\mathbf{z}}=\mathbf{W}^{H} \mathbf{A}^{H} \mathbf{t} .
$$

Combining the effect of ISI and noise, we can express the error $\tilde{\mathbf{e}}=\tilde{\mathbf{z}}-\tilde{\mathbf{s}}$ as

$$
\begin{aligned}
\tilde{\mathbf{e}} & =\mathbf{W}^{H}\left(\mathbf{A}^{H} \mathbf{t}-\mathbf{W} \tilde{\mathbf{s}}\right) \\
& =\mathbf{W}^{H} \mathbf{A}^{H} \mathbf{t}-\tilde{\mathbf{s}} .
\end{aligned}
$$

The mean square error at the output of the system using the widely linear equalizer is given by

$$
\begin{aligned}
\mathbf{J}(\tilde{\mathbf{e}})= & \operatorname{Tr}\left[E\left\{\tilde{\mathbf{e}} \tilde{\mathbf{e}}^{H}\right\}\right] \\
= & \operatorname{Tr}\left[\mathbf{W}^{H} \mathbf{A}^{H} E\left\{\mathbf{t t}^{H}\right\} \mathbf{A W}-\mathbf{W}^{H} \mathbf{A}^{H} E\left\{\tilde{\mathbf{t s}}^{H}\right\}\right. \\
& \left.-E\left\{\tilde{\mathbf{s t}}^{H}\right\} \mathbf{A W}-E\left\{\tilde{\mathbf{s}} \tilde{\mathbf{S}}^{H}\right\}\right] \\
= & \operatorname{Tr}\left[\mathbf { W } ^ { H } \left(\mathbf{A}^{H} \mathbf{C}_{\mathbf{t t}} \mathbf{A}-\mathbf{A}^{H} \mathbf{C}_{\mathbf{t s}}-\mathbf{C}_{\mathbf{s t}} \mathbf{A}\right.\right. \\
& \left.\left.+\mathbf{I}_{N}\right) \mathbf{W}\right] .
\end{aligned}
$$

Replacing A by (11), the MSE for all $N$ block symbols is given by

$$
\mathbf{J}(\tilde{\mathbf{e}})=\frac{1}{N} \operatorname{Tr}\left[\left(\mathbf{I}_{N}-\mathbf{C}_{\mathbf{t s}}^{\mathbf{H}} \mathbf{C}_{\mathbf{t t}}^{-\mathbf{1}} \mathbf{C}_{\mathbf{t s}}\right)\right] .
$$

Rewriting $\mathbf{J}(\tilde{\mathbf{e}})$ as a function of the frequency response of the channels we have

$$
\begin{aligned}
\mathbf{J}(\tilde{\mathbf{e}})= & \frac{1}{N} \operatorname{Tr}\left\{\left[\sigma_{n}^{2}\left(\mathbf{H}_{\text {mod }}+\mathbf{H i}_{\text {mod }}+\sigma_{n}^{2} \mathbf{I}_{N}\right)+\boldsymbol{\Lambda}\right]^{-1}\right. \\
& \left.\times\left[\sigma_{n}^{2}\left(\mathbf{H i}_{\text {mod }}+\sigma_{n}^{2} \mathbf{I}_{N}\right)\right]\right\},
\end{aligned}
$$

where $\Lambda, \mathbf{H}_{\text {mod }}$ and $\mathbf{H i}_{\text {mod }}$ are expressed, respectively, as

$$
\begin{gathered}
\boldsymbol{\Lambda}=\sigma_{i}^{2}\left(\mathbf{H H}^{H} \mathbf{U} \sum_{k=0}^{K} \mathbf{H}_{i k} \mathbf{H}_{i k}^{H} \mathbf{U}+\mathbf{U} \mathbf{H} \mathbf{H}^{H} \mathbf{U} \sum_{k=0}^{K} \mathbf{H}_{i k} \mathbf{H}_{i k}^{H}\right. \\
\left.-\mathbf{H}^{*} \mathbf{U H}^{H} \sum_{k=0}^{K} \mathbf{H}_{i k} \mathbf{U H}_{i k}^{T}-\mathbf{H} \mathbf{U} \mathbf{H}^{T} \sum_{k=0}^{K} \mathbf{H}_{i k}^{*} \mathbf{U} \mathbf{H}_{i k}^{H}\right) \\
\mathbf{H}_{m o d}=\mathbf{H} \mathbf{H}^{H}+\mathbf{U} \mathbf{H} \mathbf{H}^{H} \mathbf{U}
\end{gathered}
$$

and

$$
\mathbf{H i}_{\text {mod }}=\sigma_{i}^{2} \sum_{k=0}^{K} \mathbf{H}_{i k} \mathbf{H}_{i k}^{H}+\mathbf{U} \sum_{k=0}^{K} \mathbf{H}_{i k} \mathbf{H}_{i k}^{H} \mathbf{U} .
$$

This result may not be intuitive; however, it is possible to see that when removing the terms corresponding to the interferences we have $\mathbf{J}(\tilde{\mathbf{e}})=\frac{1}{N} \operatorname{Tr}\left[\sigma_{n}^{2}\left(\mathbf{H}_{\text {mod }}+\sigma_{n}^{2} \mathbf{I}_{N}\right)^{-1}\right]$, which is the equation corresponding to the WL-MMSE equalizer for SC-FDE system without interferences [24]. Again, considering an impartial estimate the effective SINR $\gamma_{W L-M M S E}$ after WL-MMSE equalization is given by.

$$
\gamma_{W L-M M S E}=\frac{1}{\mathbf{J}(\tilde{\mathbf{e}})}-1 .
$$

\section{SINR for the IB-DFE receivers}

In the case of the WL IB-DFE equalizer, after the fast inverse Fourier transform, the error of the estimated symbol $\tilde{\hat{\mathbf{s}}}^{l}$ (with length $N$ ) can be expressed by

$$
\tilde{\mathbf{e}}=\mathbf{W}^{H}\left(\hat{\mathbf{s}}^{l}-\mathbf{s}\right)=\mathbf{W}^{H}\left(\mathbf{A}^{l, H} \mathbf{t}+\mathbf{B}^{l, H} \hat{\mathbf{s}}^{l-1}-\mathbf{s}\right),
$$

and the mean square error $\mathbf{J}(\tilde{\mathbf{e}})=E\left\{\tilde{\mathbf{e}} \tilde{\mathbf{e}}^{H}\right\}$ can be expressed by

$$
\begin{aligned}
\mathbf{J}(\tilde{\mathbf{e}})= & \frac{1}{N} \operatorname{Tr}\left[\left(\mathbf{A}^{l, H} \mathbf{C}_{\mathbf{t t}} \mathbf{A}^{l}+\mathbf{A}^{l, H} \mathbf{C}_{\mathbf{t s}} \mathbf{B}^{l}-\mathbf{A}^{l, H} \mathbf{C}_{\mathbf{t s}}\right.\right. \\
& +\mathbf{B}^{l, H} \mathbf{C}_{\mathbf{s t}} \mathbf{A}^{l}+\mathbf{B}^{l, H} \mathbf{C}_{\mathbf{s s}} \mathbf{B}^{l}-\mathbf{B}^{l, H} \mathbf{C}_{\mathbf{s s}} \\
& \left.\left.-\mathbf{C}_{\mathbf{s t}} \mathbf{A}^{l}-\mathbf{C}_{\mathbf{s s}} \mathbf{B}^{l}+\mathbf{I}_{N}\right)\right] .
\end{aligned}
$$

Note that in the first iteration $\left(\mathbf{B}^{l}=0\right)$ the mean square error is equivalent to that of the expression 50, corresponding to the case of the regular WL-MMSE equalizer. For comparison, the mean square error $\mathbf{J}\left(\tilde{\mathbf{e}}_{\mathbf{L}}\right)$ for the IB-DFE equalizer in its SL version can be expressed by

$$
\begin{aligned}
\mathbf{J}\left(\tilde{\mathbf{e}}_{\mathbf{L}}\right)= & \frac{1}{N} \operatorname{Tr}\left[\left(\mathbf{A}_{L}^{l, H} \mathbf{C}_{\mathbf{r r}} \mathbf{A}_{L}^{l}+\mathbf{A}_{L}^{l, H} \mathbf{C}_{\mathbf{r s}} \mathbf{B}_{L}^{l}-\mathbf{A}_{L}^{l, H} \mathbf{C}_{\mathbf{r s}}\right.\right. \\
& +\mathbf{B}_{L}^{l, H} \mathbf{C}_{\mathbf{s r}} \mathbf{A}_{L}^{l}+\mathbf{B}_{L}^{l, H} \mathbf{C}_{\mathbf{s s}} \mathbf{B}_{L}^{l}-\mathbf{B}_{L}^{l, H} \mathbf{C}_{\mathbf{s s}} \\
& \left.\left.-\mathbf{C}_{\mathbf{s r}} \mathbf{A}_{L}^{l}-\mathbf{C}_{\mathbf{s s}} \mathbf{B}_{L}^{l}+\mathbf{I}_{N}\right)\right] .
\end{aligned}
$$

Thus, the SINR for the SC-FDE system using IB-DFE equalizers can be given by

$$
\gamma_{W L I B-D F E}=\frac{1}{\mathbf{J}(\tilde{\mathbf{e}})}-1
$$

and

$$
\gamma_{I B-D F E}=\frac{1}{\mathbf{J}\left(\tilde{\mathbf{e}}_{\mathbf{L}}\right)}-1 .
$$

Finally, if the Gaussian approximation is used for the ISI, the conditional BER to each channel realization can be obtained for both cases according to [25],

$$
B E R=\alpha Q \sqrt{\beta \gamma},
$$


where $\alpha$ and $\beta$ are constellation-specific parameters; $\gamma$ can be $\gamma_{M M S E}, \gamma_{W L-M M S E}, \gamma_{I B-D F E}$ or $\gamma_{W L I B-D F E}$; and $Q(x)=$ $\frac{1}{\sqrt{2 \pi}} \int_{x}^{\infty} \exp \left(\frac{t^{2}}{2}\right) d t$. The unconditional error probability is obtained by averaging over all the conditional error probabilities corresponding to the channel realizations. In general, the larger the value of $N$, the more valid the Gaussian approximation for the ISI will be.

\section{Simulation Results}

In this section, we compare the average MSE and BER obtained both with the derived analytical expressions and with numerical simulations, for SC-FDE systems using the widely linear equalizers and the SL ones. This comparison is made as a function of the number of interferences $K$ and for different values of the SIR, and aims to study and analyze the impact of the increase in the number of interferences on the behavior of the equalizers.

For these simulations, we performed the transmission of a block with size $N=128$ taking into consideration up to $K$ interferers. The information signal and the interfering signals are from a BPSK constellation $(\alpha=\beta=1)$ and the sampling frequency is $10 \mathrm{MHz}$ [26]. Both signals are affected by a quasi-static Rayleigh fading channel (invariant during block transmission time), whose model adopted was the ITU-T Vehicular A one [27]. Full knowledge of the characteristics of both the channel for the desired transmitter and the interference channels is assumed. The length of the $\mathrm{CP}$ is the minimum able to eliminate the interblock interference and the loss of energy introduced by it is taken into account in the SINR calculation. To calculate the final BER in the Monte Carlo simulations, a minimum of 400 errors were considered for each point. For $K=1$ the values considered SIR were 20,16, 13, 3 and $0 \mathrm{~dB}$; for $K=2,3$ and 4 the last two SIR values were not considered. Table I summarizes all parameters used for simulation

Tabela I: Parameters of simulation

\begin{tabular}{|l|l|}
\hline Block Size $N$ & 128 \\
\hline Modulation & BPSK \\
\hline CP size $L_{c p}$ & Enough to eliminate IBI \\
\hline Frequency of Sampling & $10 \mathrm{MHz}$ \\
\hline Type of Fading & Quasi-static \\
\hline Channel Model & Vehicular A - ITU-T \\
\hline SIR (dB) & $0,3,13,16,20$ \\
\hline
\end{tabular}

Using MMSE and WL-MMSE equalizers, Figure 3 presents Monte Carlo simulation results for a SC-FDE system under the effect of one interference. This figure presents also the BER curve obtained when we do not take into account the interferers and use the equalizer proposed in [24]. In this case, the interferers are treated as an additional noise. It is possible to see that there is a large BER reduction when using the widely linear MMSE that takes into account the interferers with respect to the one that does not, validating the approach proposed in this paper to filter together both interference and channel fading.

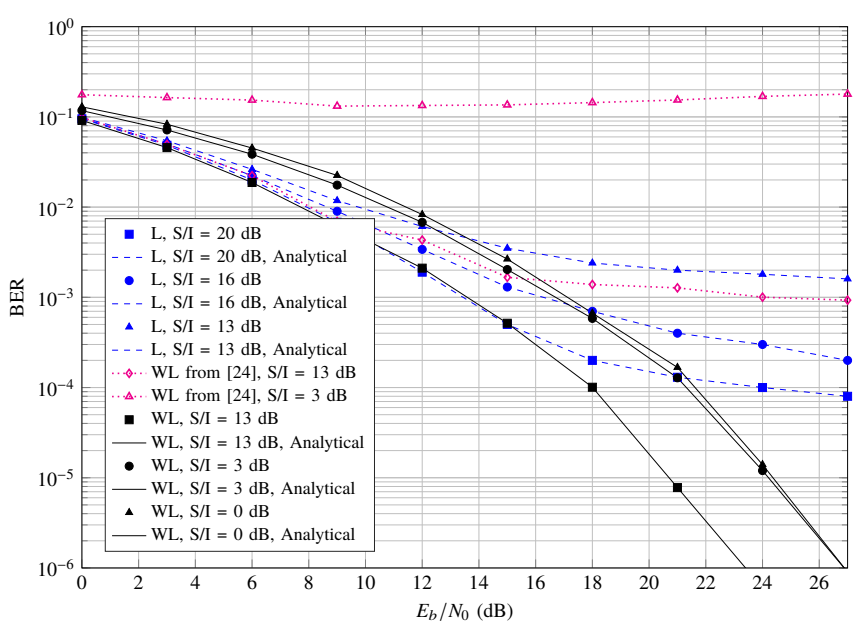

Figure 3: Error performance comparison between WL MMSE and MMSE equalizers for 1 interference.

As it was seen in [12], by using widely linear processing the receiver can drastically reduce the effect of one interference. It is possible to see that this is also true in this scenario for SC-FDE systems with a WL-MMSE equalizer: they do not show the error floor effect that appears when using the SL version. When we increase the number of interferences some interesting phenomena can be seen. Figures 4,5 and 6 now shows the BER for 2, 3 and 4 interferences respectively for the previously presented SIR values.

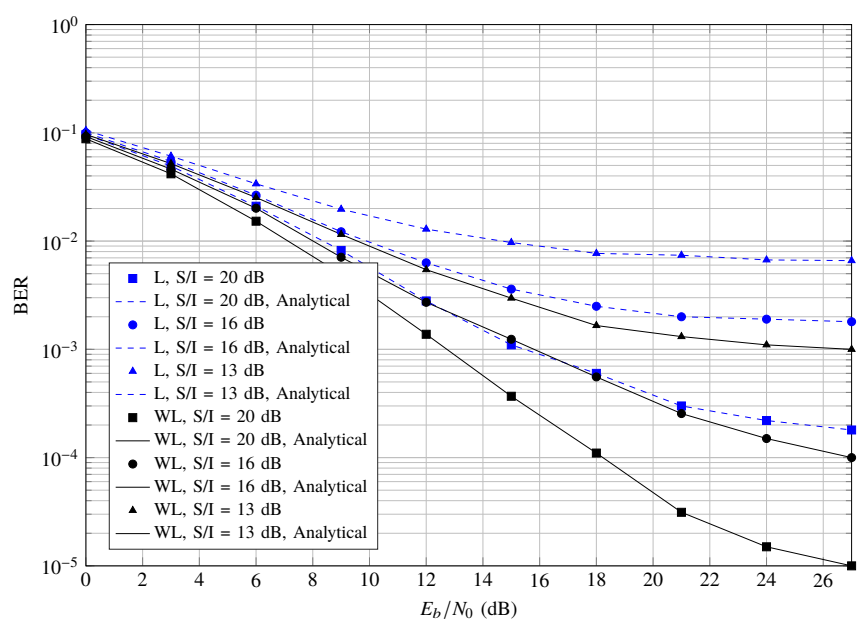

Figure 4: Error performance comparison between WL MMSE and MMSE equalizers for 2 interferences.

In the first two cases, the WL equalizer has an error performance that is now clearly sensitive to the effect of the interferences, however, it is still able to efficiently compensate for its effects, leading to a performance gain and a lower BER in relation to the SL equalizer. For the last case considered $(K=4)$, both equalizers have poor performance, as well as the fact that the WL equalizer has reached its interference cancellation threshold. Again, it should be noted that for all scenarios the simulation results match the ones obtained with the analytical expressions. 


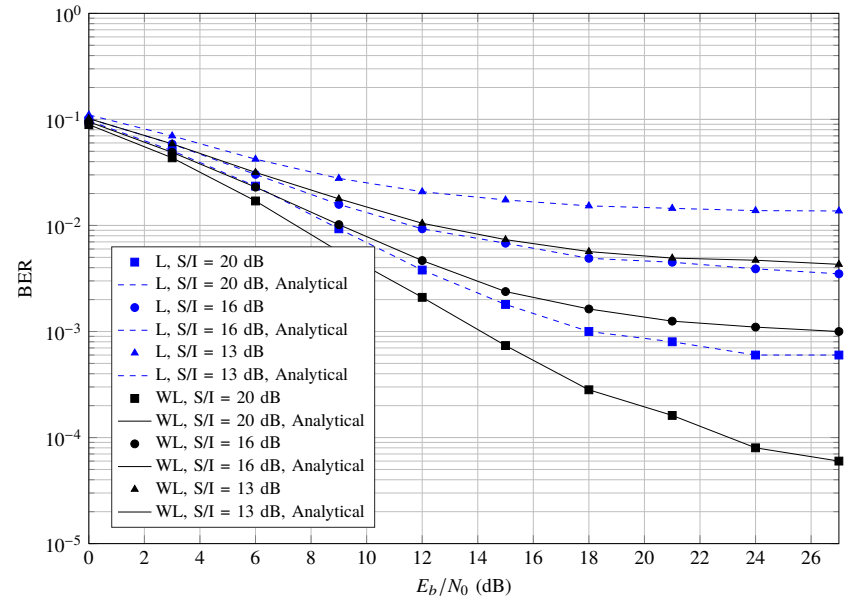

Figure 5: Error performance comparison between WL MMSE and MMSE equalizers for 3 interferences.

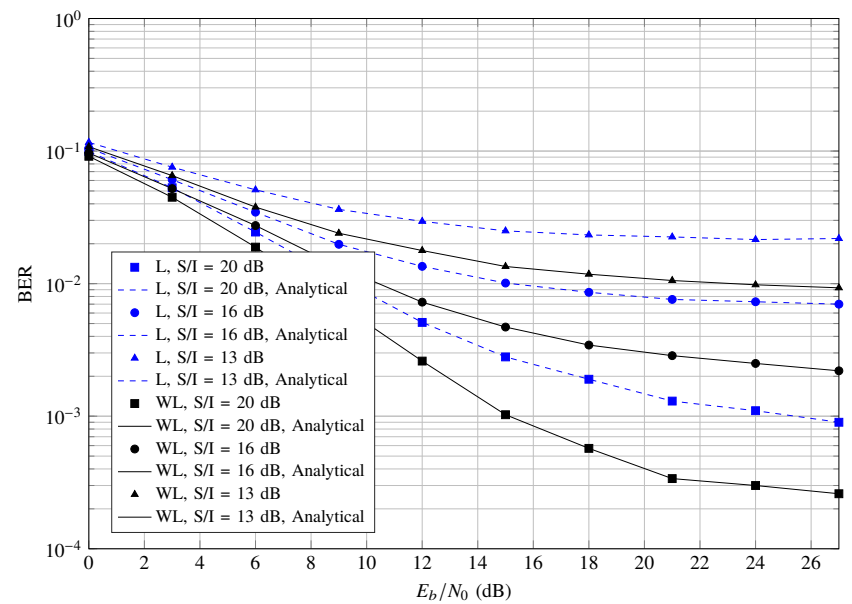

Figure 6: Error performance comparison between WL MMSE and MMSE equalizers for 4 interferences.

Concerning the proposed IB-DFE equalizer, Figure 7 shows the evolution of the widely linear equalizer at each iteration, for the case of one interferer with a SIR of $3 \mathrm{~dB}$.

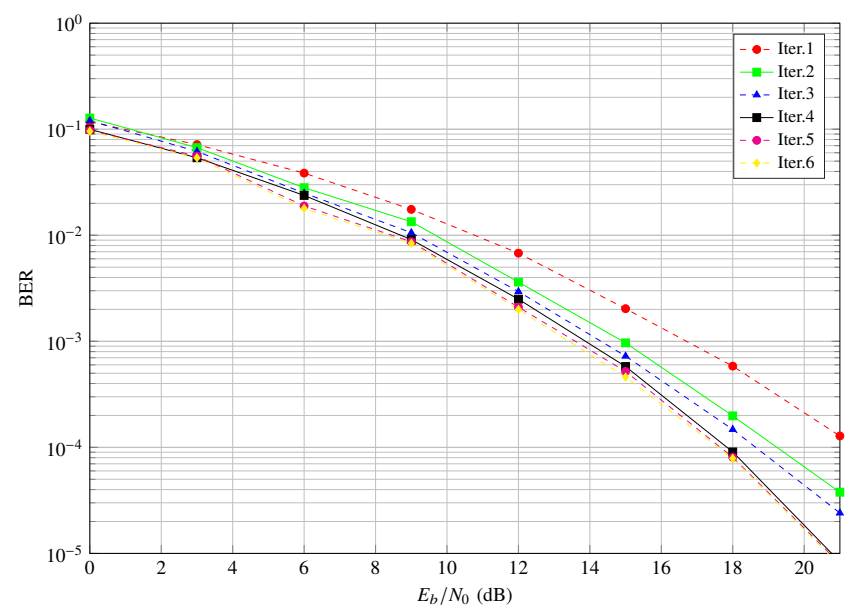

Figure 7: Evolution of the widely linear iterative equalizer.
It is worth mentioning that the first iteration corresponds to the case where the feedback filter is switched off. In the following iterations, the iterative feedback filter is taken into account. As we can note, there is a significant performance gain between the first and the fourth iterations, after which the gain is negligible. Therefore, in the following BER curves, we only show the results for the first and the fourth iterations. As we have presented the theoretical curves for the case of the WL-MMSE equalizer that corresponds to the first iteration of the WL-IB-DFE, the following figures present only their simulated results.

Figure 8 presents Monte Carlo simulation results for a SCFDE system with one interference for SIR values of 13, 3, and $0 \mathrm{~dB}$. Note, also, a performance gain when comparing the proposed widely linear iterative structure with the noniterative one (i. e., the first iteration) for all SIR values.

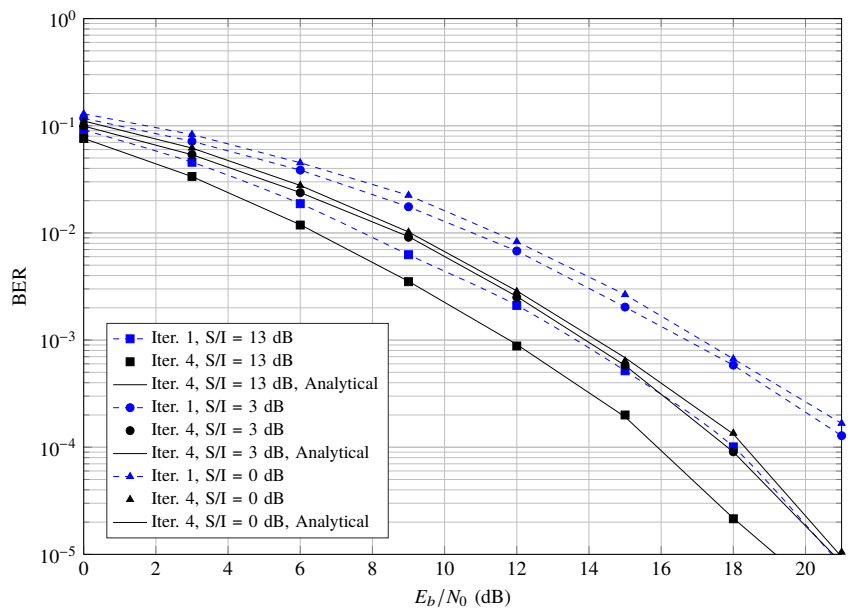

Figure 8: Error performance comparison between WL equalizers for $N=128$, the Vehicular A channel model and 1 interference.

Figures 9, 10 and 11 present BER curves for 2, 3 and 4 interferers, respectively, for the same scenario considered above.

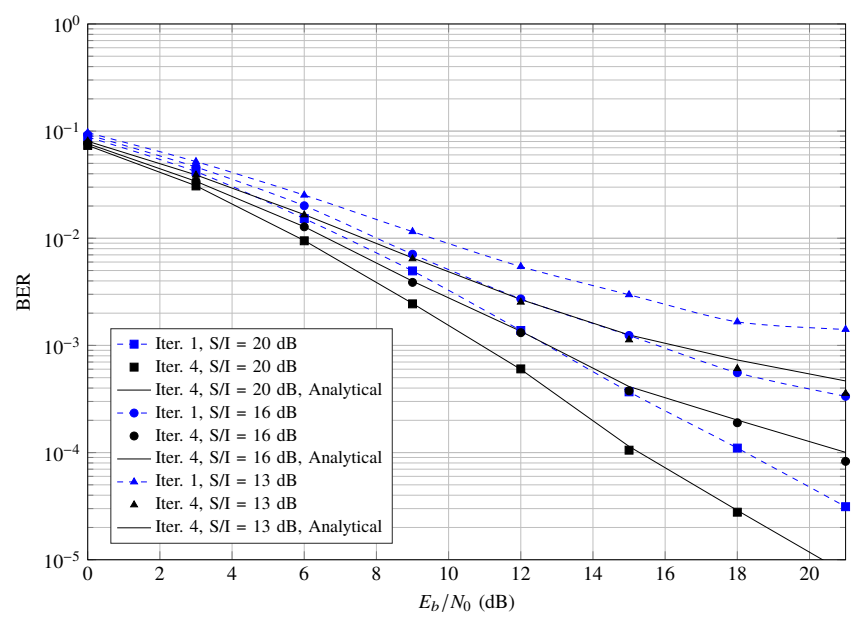

Figure 9: Error performance comparison between WL equalizers for $N=128$, the Vehicular A channel model and 2 interferences. 
It is possible to see that for low SNRs, the performance is almost the same, but as the SNR increases the widely linear iterative equalizer outperforms the non-iterative version for all SIR values. Nevertheless, the iterative structure reaches its cancellation limit at $K=4$, like the non-iterative equalizer. Thus, in all cases, the WL iterative equalizer presents a better error performance when compared to the non-iterative structure. Therefore, the proposed approach to this equalizer to compensate for interference (ISI/CCI) is validated.

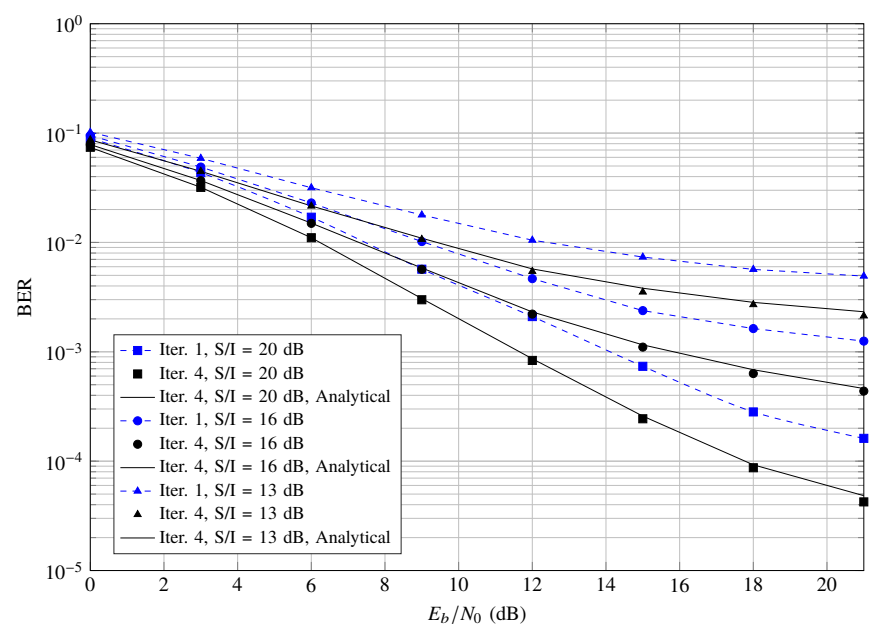

Figure 10: Error performance comparison between WL equalizers for $N=128$, the Vehicular A channel model and 3 interferences.

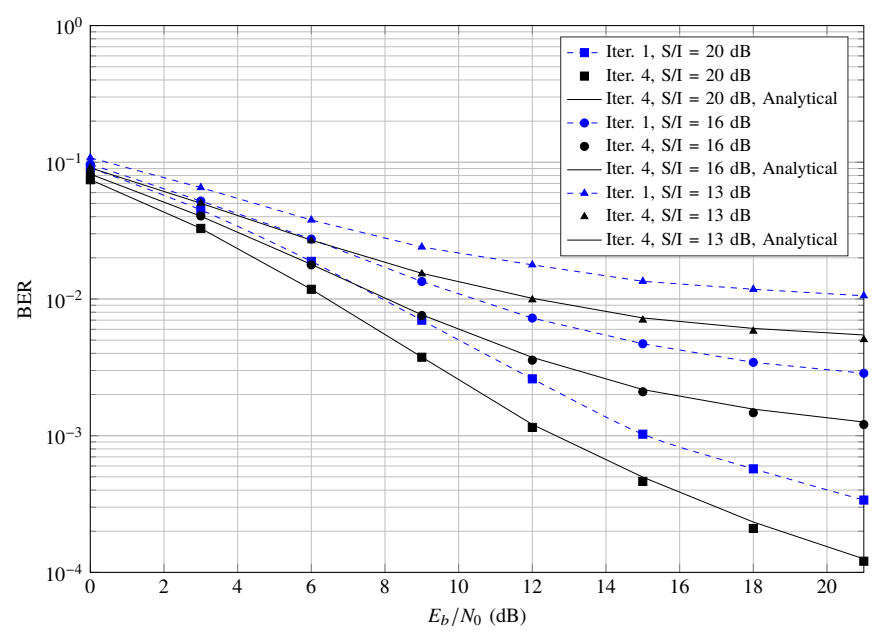

Figure 11: Error performance comparison between WL equalizers for $N=128$, the Vehicular A channel model and 4 interferences.

Again, it should be noted that for all interference values and their respective power values, the simulation results correspond to the theoretical ones. Thus, also we validate the theoretical expressions derived in section IIII, so that we can obtain the expected performance of the system in the interferences cancellation and analyze their characteristics.

It is also necessary to analyze the performance of the WL IB-DFE equalizer when compared to its SL version. Thus, the Figures 12, 13, 14 and 15 present the BER curves of the proposed equalizer, comparing it with its strictly linear version (i. e., IB-DFE) for $1,2,3$ and 4 interferences, respectively. The SIR values are the same as those for the previous simulations and again, because there is no significant improvement in performance, we present the error performance of both equalizers using four iterations.

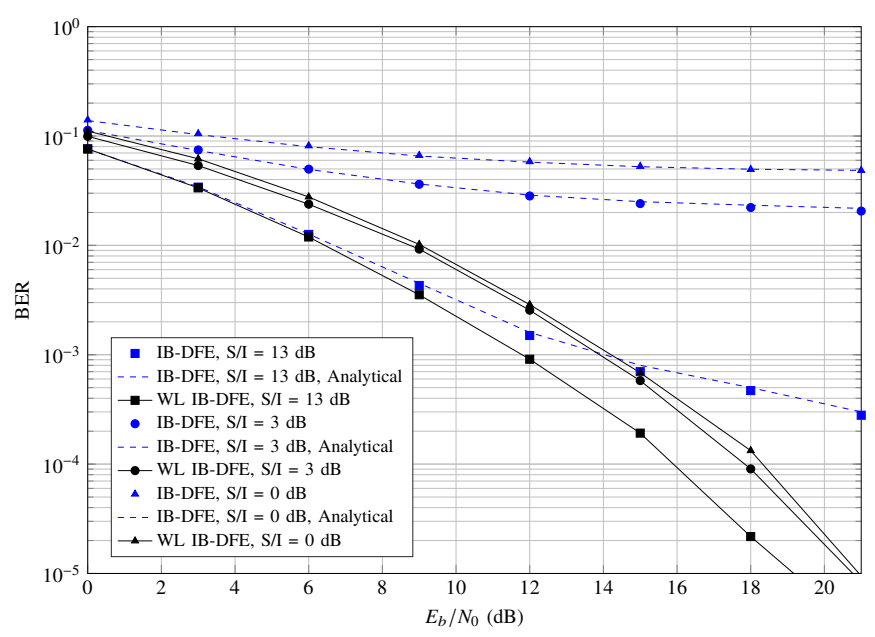

Figure 12: Error performance comparison between IB-DFE for $N=$ 128, the Vehicular A channel model and 1 interference.

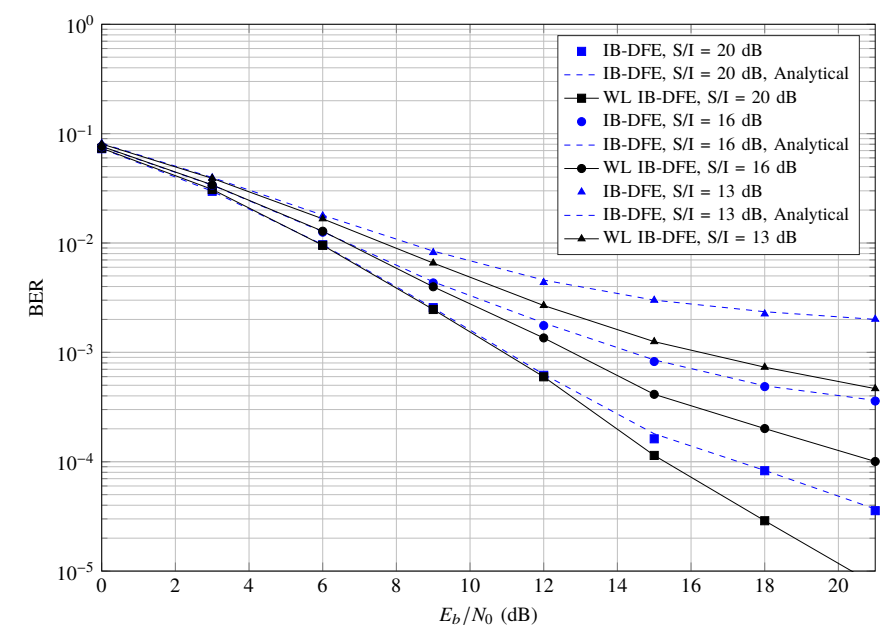

Figure 13: Error performance comparison between IB-DFE for $N=$ 128, the Vehicular A channel model and 2 interferences.

It is observed that in all cases the performance of the WL equalizer exceeds that obtained with the regular IB-DFE, especially when the SNR is high. In the case of one interferer it can be seen that the SL equalizer cannot reduce the effect of the interference, presenting a clearly inferior performance with respect to the WL IB-DFE, with an error floor for intermediate SNR values. However, for 2 to 4 interferers the performance advantage of the WL IB-DFE with respect to the linear IB-DFE is lower than the one obtained with only one interference. It is also noted that for $K=4$, the results in terms of BER of the IB-DFE equalizer resemble the results of the WL-MMSE equalizer. 


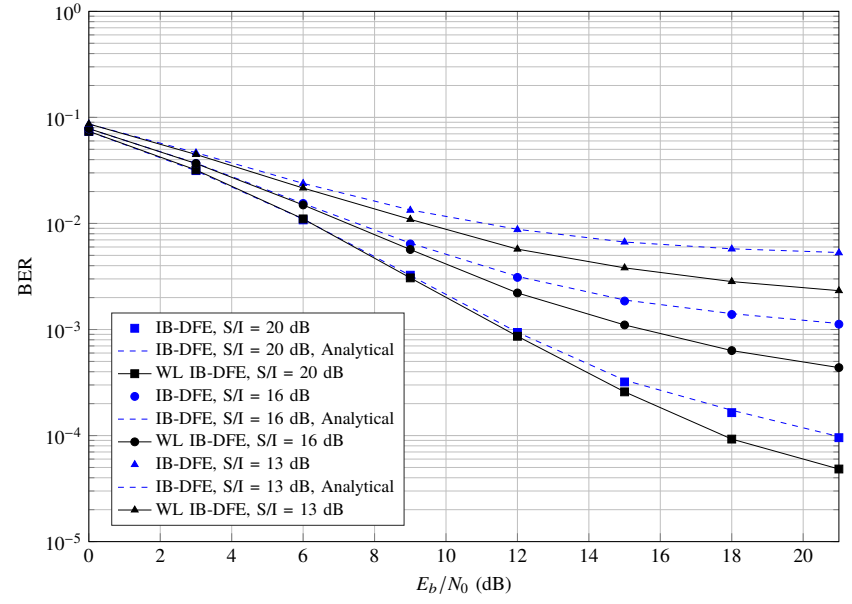

Figure 14: Error performance comparison between IB-DFE for $N=$ 128, the Vehicular A channel model and 3 interferences.

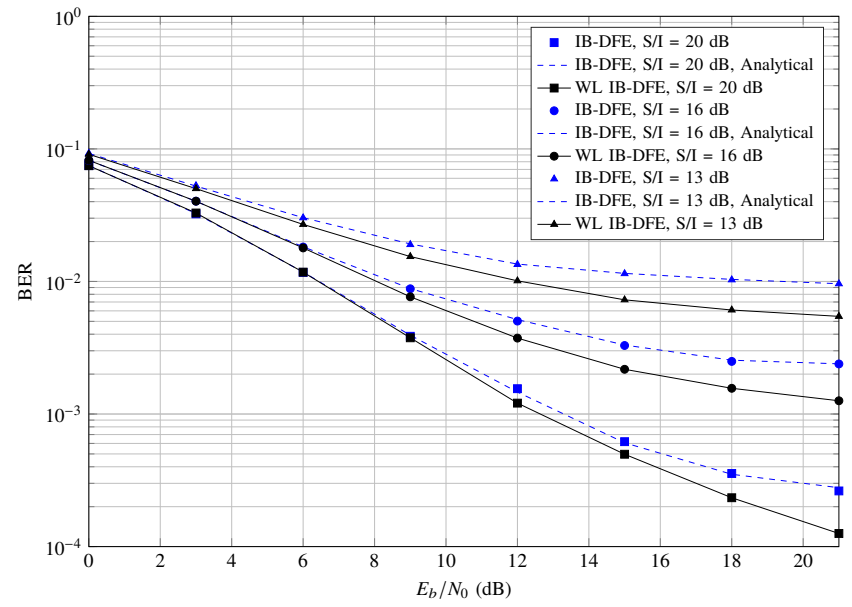

Figure 15: Error performance comparison between IB-DFE for $N=$ 128, the Vehicular A channel model and 4 interferences.

Finally, Figure 16 presents the MSE for all the presented equalizers with respect to the number of interferences, with fixed values for $E_{b} / N_{0}$ and SIR at 20 and $16 \mathrm{~dB}$, respectively.

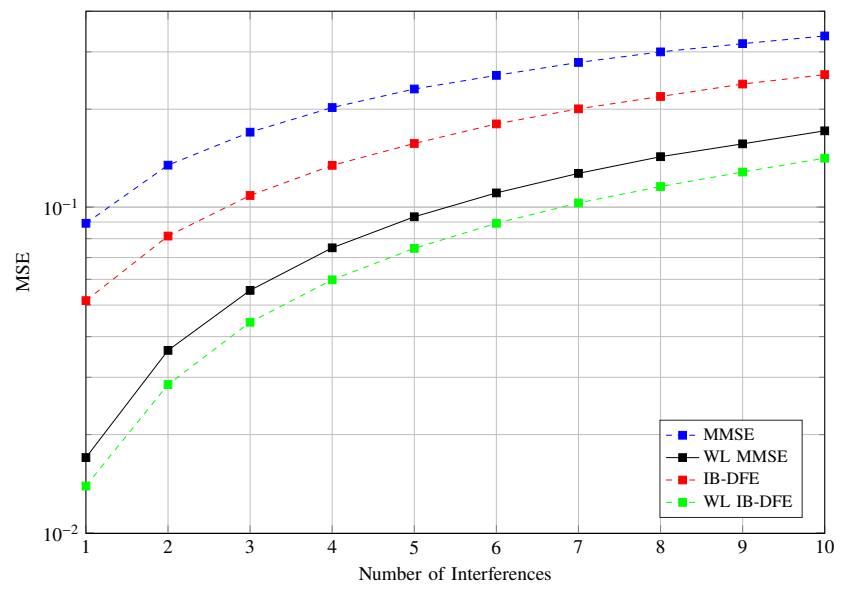

Figure 16: MSE performance versus number of interferences.
The presented MSE results were calculated with [46, (52), (58) and (59), which were validated previously. We can see a large advantage for the WL equalizers throughout the range of values: as an example, the MMSE equalizer with 1 interference has similar performance as the WL-MMSE one with 5 interferences. Likewise, for 2 interferences the performance of MMSE equalizer is the same as the one from a widely linear equalizer under the effect of 8 interferences. Analyzing the iterative equalizers, we have that the WL IBDFE equalizer with 6 interferences has the same mean square error of the MMSE equalizer with 1 interference. As well as the same with 8 interferences has approximate performance with its SL version with 3 interferences. As expected, as the number of interferences increases, the WL equalizer gain relative to its linear version approaches $3 \mathrm{~dB}$ [11], [28].

\section{Conclusion}

We have presented in this paper analytical expressions for the MSE and BER for a SC-FDE system with widely linear equalizers in regular and iterative versions designed to cope with $K$ interferences. It was found out that the results obtained with the derived expressions correspond to the ones obtained by simulation, and they show that regardless of the number of interferences the WL equalizers will have a better error performance when compared to the linear ones. A suggestion for future work is an expansion of the analysis presented for a scenario in which channel estimation errors are considered, and the usage of multiple antennas in the receiver for interference cancellation.

\section{ACKNOWLEDGMENT}

The authors acknowledge the financial support from the scholarships from National Council for the Improvement of Higher Education (Capes).

\section{REFERENCES}

[1] D. Falconer, S. L. Ariyavisitakul, A. Benyamin-Seeyar, and B. Eidson, "Frequency domain equalization for single-carrier broadband wireless systems," IEEE Communications Magazine, vol. 40, no. 4, pp. 58-66, 2002, doi:10.1109/35.995852.

[2] A. Ali, W. Dong, L. Renfa, and E. Eldesouky, "Isi and papr immune ieee $802.11 \mathrm{p}$ channels based on single-carrier frequency domain equalizer." KSII Transactions on Internet \& Information Systems, vol. 10, no. 11, 2016, doi:10.3837/tiis.2016.11.017.

[3] W. Nam, D. Bai, J. Lee, and I. Kang, "Advanced interference management for $5 \mathrm{~g}$ cellular networks," IEEE Communications Magazine, vol. 52, no. 5, pp. 52-60, 2014, doi:10.1109/MCOM.2014.6815893.

[4] O. Popescu and D. C. Popescu, "On the performance of sub-band precoded ofdm in the presence of narrowband co-channel interference," IEEE Transactions on Broadcasting, vol. 62, no. 3, pp. 736-743, 2016, doi:10.1109/TBC.2016.2570004.

[5] S. Kumar, F. Kaltenberger, A. Ramirez, and B. Kloiber, "Robust ofdm diversity receiver under co-channel narrowband interference," in 2018 14th International Conference on Wireless and Mobile Computing, Networking and Communications (WiMob). IEEE, 2018, pp. 1-8, doi:10.1109/WiMOB.2018.8589166.

[6] J. Proakis, Digital Communications, 4th ed. McGrawHill, 2000.

[7] N. Benvenuto and S. Tomasin, "On the comparison between ofdm and single carrier modulation with a dfe using a frequency-domain feedforward filter," IEEE Transactions on Communications, vol. 50, no. 6, pp. 947-955, 2002, doi:10.1109/TCOMM.2002.1010614. 
[8] M. Noune and A. Nix, "Frequency-domain precoding for single carrier frequency-division multiple access," IEEE Commun. Mag, vol. 47, no. 6, pp. 68-74, 2009, doi:10.1109/MCOM.2009.5116802.

[9] N. Hajiabdolrahim and M. Sabbaghian, "Analysis of the impact of the receiver i/q imbalance on the achievable rate of sc-fde systems," IEEE Transactions on Vehicular Technology, vol. 67, no. 6, pp. 5530-5534, 2018, doi:10.1109/TVT.2017.2785345.

[10] N. Souto, R. Dinis, A. Correia, and C. Reis, "Interference-aware iterative block decision feedback equalizer for single-carrier transmission," IEEE Transactions on Vehicular Technology, vol. 64, no. 7, pp. 33163321, 2015, doi:10.1109/TVT.2014.2352266

[11] B. Picinbono and P. Chevalier, "Widely linear estimation with complex data," IEEE transactions on Signal Processing, vol. 43, no. 8, pp. 2030-2033, 1995, doi:10.1109/78.403373.

[12] P. Chevalier and F. Pipon, "New insights into optimal widely linear array receivers for the demodulation of bpsk, msk, and gmsk signals corrupted by noncircular interferences-application to saic," IEEE Transactions on Signal Processing, vol. 54, no. 3, pp. 870-883, 2006, doi:10.1109/TSP.2005.862946.

[13] P. Chevalier, R. Chauvat, and J.-P. Delmas, "Quasi-rectilinear (msk, gmsk, oqam) co-channel interference mitigation by three inputs widely linear fresh filtering," in 2015 IEEE International Conference on Acoustics, Speech and Signal Processing (ICASSP). IEEE, 2015, pp. 2434-2438, doi:10.1109/ICASSP.2015.7178408.

[14] $\_$, "Enhanced widely linear filtering to make quasi-rectilinear signals almost equivalent to rectilinear ones for saic/maic," IEEE Transactions on Signal Processing, vol. 66, no. 6, pp. 1438-1453, 2018, doi:10.1109/TSP.2017.2784403.

[15] H. Fhima, R. Zayani, H. Shaïek, D. Roviras, B. S. Chang, and R. Bouallegue, "Widely linear equalizer performance with multiple independent interferences," in Computers and Communications (ISCC), 2017 IEEE Symposium on. IEEE, 2017, pp. 912-917, doi:10.1109/ISCC.2017.8024642.

[16] H. Fhima, H. Shaïek, R. Zayani, D. Roviras, B. S. Chang, and R. Bouallegue, "Analysis of widely linear equalization over frequency selective channels with multiple interferences," in 2018 14th International Conference on Wireless and Mobile Computing, Networking and Communications (WiMob). IEEE, 2018, pp. 83-88, doi:10.1109/WiMOB.2018.8589138.

[17] M. Kim, J. H. Cho, and J. S. Lehnert, "Asymptotically optimal lowcomplexity sc-fde with noise prediction in data-like improper-complex interference," IEEE Transactions on Wireless Communications, vol. 15, no. 3, pp. 2090-2103, 2016, doi:10.1109/TWC.2015.2498604.

[18] B. S. Chang, C. A. da Rocha, D. Le Ruyet, and D. Roviras, "Widely linear iterative equalizers for sc-fde systems," in Wireless Communication Systems (ISWCS 2013), Proceedings of the Tenth International Symposium on. VDE, 2013, pp. 1-5.

[19] B. S. Chang, C. A. da Rocha, L. S. Resende, and D. Le Ruyet, "On the effect of channel estimation errors in sc-fde systems using widely linear iterative equalization," in Wireless Communication Systems (ISWCS), 2015 International Symposium on. IEEE, 2015, pp. 526530, doi:10.1109/ISWCS.2015.7454400.

[20] B. S. Chang, C. A. da Rocha, H. Fhima, R. Zayani, H. Shaiek, and D. Roviras, "On the performance of a widely linear sc-fde system under multiple independent interferences," in Personal, Indoor, and Mobile Radio Communications (PIMRC), 2017 IEEE 28th Annual International Symposium on. IEEE, 2017, pp. 1-5, doi:10.1109/PIMRC.2017.8292380.

[21] R. P. Junior, C. A. da Rocha, and B. S. Chang, "Multiple independent interferences cancellation for sc-fde systems using widely linear iterative equalizers," in 2018 IEEE 10th Latin-American Conference on Communications (LATINCOM). IEEE, 2018, pp. 1-6, doi:10.1109/LATINCOM.2018.8613234.

[22] N. Benvenuto and S. Tomasin, "Iterative design and detection of a dfe in the frequency domain," IEEE Transactions on Communications, vol. 53, no. 11, pp. 1867-1875, 2005, doi:10.1109/TCOMM.2005.858666.

[23] J. Cioffi, "379a class note: Signal processing and detection," 2008.

[24] B. S. Chang, C. A. Da Rocha, D. Le Ruyet, and D. Roviras, "Widely linear mmse precoding and equalization techniques for sc-fde systems," EURASIP Journal on Advances in Signal Processing, vol. 2014, no. 1, p. 124, 2014, doi:10.1186/1687-6180-2014-124.

[25] G. L. Stüber, Principles of mobile communication. Springer, 2017, vol. 3.

[26] R. Zakaria and D. Le Ruyet, "A novel filter-bank multicarrier scheme to mitigate the intrinsic interference: Application to mimo systems," IEEE Transactions on Wireless Communications, vol. 11, no. 3, pp. 1112-1123, 2012, doi:10.1109/TWC.2012.012412.110607.

[27] E. U. T. R. Access, "Base station (bs) radio transmission and reception," 3GPP TS, vol. 36, p. V9, 2009.

[28] A. Lampe and M. Breiling, "Asymptotic analysis of widely linear mmse multiuser detection-complex vs real modulation," in Information Theory Workshop, 2001. Proceedings. 2001 IEEE. IEEE, 2001, pp. 55-57, doi:10.1109/ITW.2001.955134.

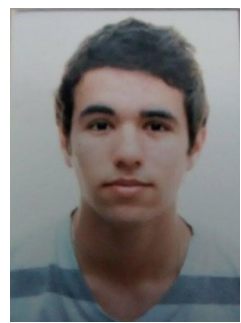

Rogério Pereira Junior was born in São José, Santa Catarina, Brazil, in 1994. He holds a degree in Telecommunications Systems from the Federal Institute of Santa Catarina (IFSC), Brazil in 2016, and received his M.Sc. in electrical engineering from the Federal University of Santa Catarina (UFSC), Florianópolis, Brazil, in 2018. His research interests include signal processing for digital communications, widely linear processing, equalization and multi-carrier systems. He is pursuing the $\mathrm{Ph} . \mathrm{D}$. degree in electrical engineering at the

Federal University of Santa Catarina.

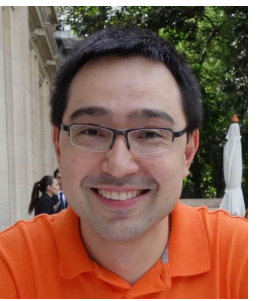

Bruno Sens Chang was born in Curitiba, Paraná, Brazil, in 1984. He received the B.Sc. Degree in Telecommunications Engineering from the Regional University of Blumenau (FURB), Brazil, in 2006, the M.Sc. degree in electrical engineering from the Federal University of Santa Catarina (UFSC), Florianópolis, Brazil, in 2008, and the D.Sc. degree in electrical engineering from the Federal University of Santa Catarina (UFSC), Florianópolis, Brazil, and the Conservatoire National des Arts et Métiers (CNAM), Paris, France in 2012. He was a visiting professor at the CEDRIC laboratory at CNAM in 2018. He is currently an Assistant Professor at the Federal University of Technology - Paraná (UTFPR), Curitiba, Brazil. His research interests include multi-carrier systems, widely linear processing and energy efficient communications.

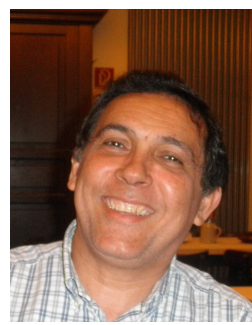

Carlos Aurélio Faria da Rocha was born in Santarém, Pará, Brazil, in July 1956. He received the M.Sc. degree in electrical engineering from the Federal University of Santa Catarina (UFSC), Santa Catarina, Brazil, in 1986, and the Ph.D. degree from the State University of Campinas, São Paulo, Brazil, in 1996. From 1993 to 1994, he was with Laboratoire des Signaux et Systèmes, SUPELEC, Gif-Sur-Yvette, France. From 2008 to 2009, he was with the Conservatoire National des Arts et Métiers, Laboratoire Cedric Traitement du Signal et Architectures Électroniques, Paris, France. Since 1983, he has been with the Electrical Engineering Department, UFSC, where he is currently a Full Professor. His current research interests include multicarrier communication and widely linear signal processing. 